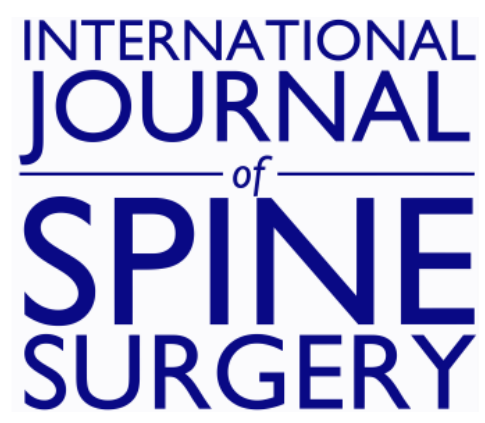

\title{
A Narrative Review of Uniportal Endoscopic Lumbar Interbody Fusion: Comparison of Uniportal Facet-Preserving Trans-Kambin Endoscopic Fusion and Uniportal Facet-Sacrificing Posterolateral Transforaminal Lumbar Interbody Fusion
}

Hyeun Sung Kim, Pang Hung Wu, Koichi Sairyo and Il-Tae Jang

Int J Spine Surg 2021, 15 (suppl 3) S72-S83

doi: https://doi.org/10.14444/8166

http://ijssurgery.com/content/15/suppl_3/S72

This information is current as of April 26, 2023.

Email Alerts Receive free email-alerts when new articles cite this article. Sign up at: http://ijssurgery.com/alerts 


\title{
A Narrative Review of Uniportal Endoscopic Lumbar Interbody Fusion: Comparison of Uniportal Facet- Preserving Trans-Kambin Endoscopic Fusion and Uniportal Facet-Sacrificing Posterolateral Transforaminal Lumbar Interbody Fusion
}

\author{
HYEUN SUNG KIM, MD, PHD ${ }^{1}$; PANG HUNG WU, MBBS, FRCS ${ }^{1,2}$; KOICHI SAIRYO, MD, PHD ${ }^{3}$; AND \\ IL-TAE JANG, MD, PHD ${ }^{1}$ \\ ${ }^{I}$ Nanoori Gangnam Hospital, Seoul, South Korea; ${ }^{2}$ National University Health Systems, Singapore, Singapore; ${ }^{3}$ Department of Orthopedics, Tokushima University \\ Graduate School, Tokushima, Japan
}

\begin{abstract}
Uniportal endoscopic lumbar interbody fusion aims to achieve the bony union of 2 lumbar segments through cage insertion using full spinal endoscopy. Endoscopic fusion can adjust foraminal height and disc height, improve alignment, and minimize collateral soft tissue damage during the insertion of an interbody cage. The surgery is performed under constant irrigation with normal saline and an optical endoscopic lens close to the targeted disc segment. Two main subtypes of uniportal endoscopic fusion are currently described in the literature. We broadly classify them into facet-preserving and facet-sacrificing endoscopic lumbar interbody fusions. We have termed them uniportal facet-preserving trans-Kambin endoscopic fusion and uniportal facetsacrificing posterolateral transforaminal lumbar interbody fusion. In this article, we review the current literature and discuss the history, indications, contraindications, technical differences, clinical outcomes, and complications of uniportal endoscopic interbody fusion surgery.
\end{abstract}

Special Issue

Keywords: degenerative spinal disease, endoscopic spine surgery, endoscopic fusion, spinal fusion, novel spine techniques, transforaminal lumbar interbody fusion, spinal endoscopy

\section{INTRODUCTION}

Endoscopic spine surgery is gaining traction as a subspecialist practice among spine surgeons. It provides direct and targeted access to the disc space with limited soft tissue trauma. Endoscopic fusion is an extended indication of lumbar spinal endoscopy. Endoscopic spinal fusion is made possible by advancements in endoscopic optical technology, improved endoscopic instruments to facilitate soft and bony tissue removal, and improved surgical techniques for endoscopic discectomy and decompression.

There are 2 distinct and well-defined anatomical corridors through which equipment can safely pass to reach a targeted end plate for fusion: the transforaminal corridor and the posterolateral corridor. For easy differentiation between the 2 subtypes of uniportal full endoscopic interbody fusion, we describe the first subtype as uniportal facet-preserving trans-Kambin endoscopic fusion (Figure 1) and the second subtype as uniportal facet-sacrificing posterolateral endoscopic fusion. (Figure 2).

Early endoscopic surgeries adopted the target working zone of Kambin's triangle during transforaminal endoscopic lumbar discectomy. The use of this working corridor to perform endoscopic surgeries, including fusion, gave rise to the development of uniportal facet-preserving trans-Kambin endoscopic fusion. ${ }^{1,2}$

With the evolution of endoscopic spine surgery, larger working channel endoscopes and corresponding endoscopic drills and instruments designed for lumbar endoscopic unilateral laminotomy for bilateral decompression were developed. Uniportal facetsacrificing posterolateral transforaminal lumbar interbody fusion was subsequently described as a means of performing interbody fusion using a largechannel endoscope with the facet removed to create working space for disc preparation and insertion of the interbody cage. ${ }^{3,4}$ 

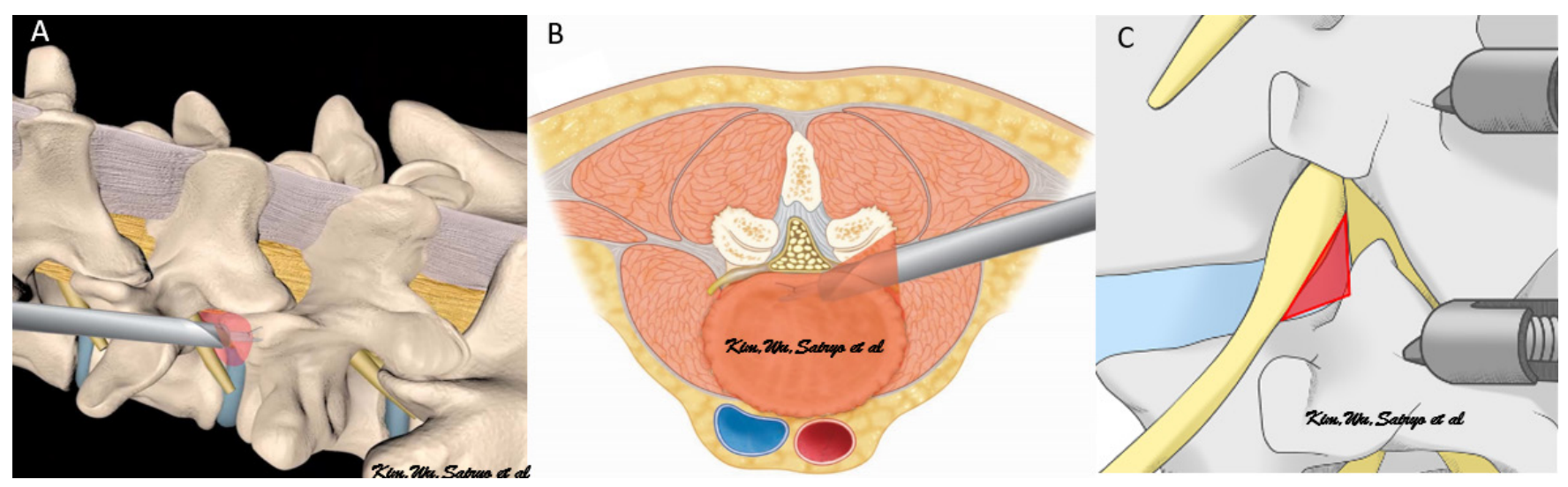

Figure 1. Illustration of uniportal facet-preserving trans-Kambin endoscopic fusion. (A) Oblique view of the lumbar spine model shows left L4/5 uniportal facetpreserving trans-Kambin endoscopic fusion. Note the red Kambin's triangle with/without foraminoplasty for gaining access to the disc space. (B) Axial view shows left L4/5 uniportal facet-preserving trans-Kambin endoscopic fusion. Note the tangerine-colored Kambin's triangle for gaining access to the disc space for cage insertion. (C) Oblique magnified view of lumbar spine shows left L4/5 Kambin's triangle in red, which is subtended by the exiting nerve root as the hypotenuse, the facet joint as the opposite side, and the proximal vertebral end plate of the caudal vertebral body as the adjacent side. This anatomical corridor is a natural window of safe access to the disc, with no significant neurovascular structures within the triangle. The size of the triangle varies according to pathological anatomy.

\section{EVOLUTION OF UNIPORTAL ENDOSCOPIC INTERBODY FUSION}

\section{Uniportal Facet-Preserving Trans-Kambin Endoscopic Fusion}

Leu and Hauser first described percutaneous endoscopic lumbar interbody fusion in 1997..$^{5}$ A similar technique was discussed by Osman. ${ }^{6}$ In the original descriptions of endoscopic lumbar interbody fusion, a biportal endoscopic technique was performed. The viewing portal was placed on the contralateral side of the cage insertion portal. In a left lumbar percutaneous endoscopic lumbar interbody fusion, an arthroscope was inserted on the right side of the intended level, parallel to the disc, through the right Kambin's triangle with fluoroscopic guidance. The arthroscope guided the disc preparation, which was done from the left Kambin's triangle. Guidewire was inserted from the left side, followed by serial dilation with reamers and disc shavers using endoscopic visualization of the right contralateral side. The endoscopic visualization was helpful in end plate preparation. However, the neural elements were not visualized during the disc preparation and bone graft insertion, and the procedure relied heavily on motor-evoked potentials and electromyograms to monitor the myogenic responses.

As endoscopic techniques became more refined, both technological and technical advancements were
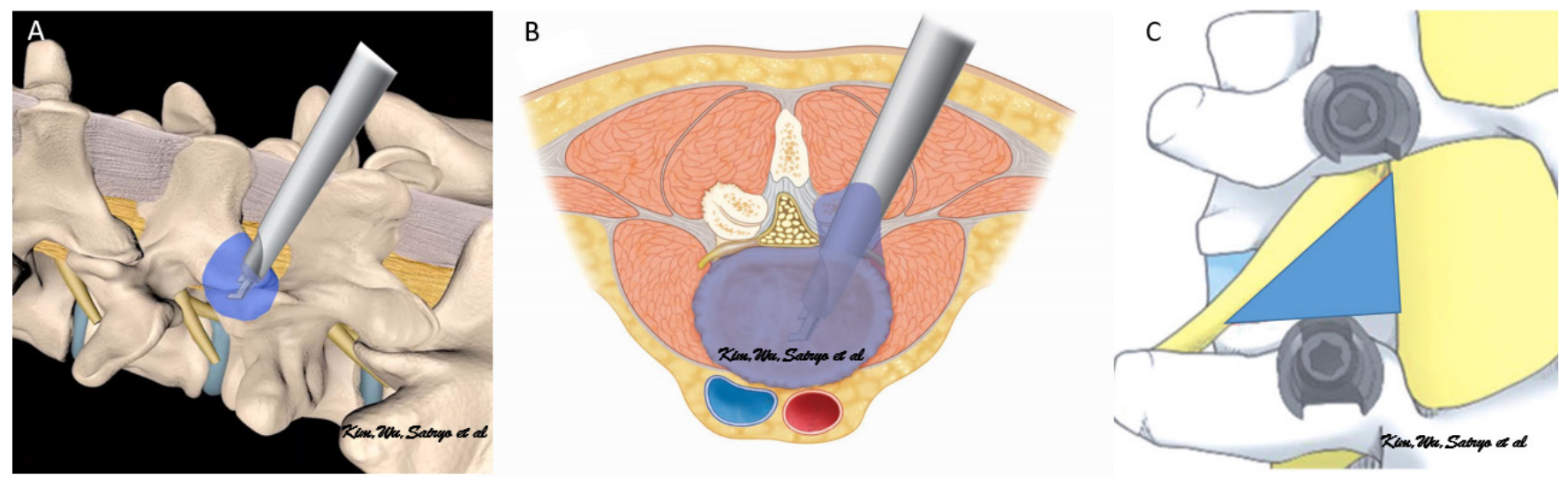

Figure 2. Illustration of uniportal facet-sacrificing posterolateral endoscopic fusion. (A) Oblique view of the lumbar spine model shows left L4/5 uniportal facetsacrificing posterolateral transforaminal lumbar interbody fusion. Note that the blue facet joint was removed to gain access to the disc space. (B) Axial view shows left L4/5 uniportal facet-sacrificing posterolateral transforaminal lumbar interbody fusion. Note the blue facetectomy and extension into the disc space, demonstrating the working corridor and access in the uniportal facet-sacrificing posterolateral transforaminal lumbar interbody fusion approach. (C) Oblique magnified view of the lumbar spine shows left L4/5 extended Kambin's triangle in blue, which is subtended by the exiting nerve root as the hypotenuse, the traversing nerve root as the opposite side, and the proximal vertebral end plate of the caudal vertebral body as the adjacent side. This anatomical corridor is a natural window of safe access to the disc, with no significant neurovascular structures within the triangle. The size of the extended Kambin's triangle is less dependent on the pathology of the facet joint. It is bigger in lower lumbar segments than in upper lumbar segments. 
made in the transforaminal route. A number of pioneers of the trans-Kambin approach to endoscopic fusion described their technique as using the same port of entry for the endoscope and cage insertion through Kambin's triangle. Morgenstern et al described uniportal endoscopic trans-Kambin lumbar interbody fusion with a transforaminal endoscope. The authors docked at Kambin's triangle, performed the foraminoplasty and disc preparation, and inserted the expandable cage, followed by pedicle screws insertion. ${ }^{7}$ Wang and Grossman described a similar technique in which they used a transforaminal endoscope with an 8-mm outer diameter and performed fusion with an expandable OptiMesh cage through the same anatomical corridor. ${ }^{8}$ Standalone uniportal facet-preserving trans-Kambin endoscopic fusion without pedicle screws was described by Lewandrowski et al as LEW-LIF, with the majority of patients reporting favorable outcomes. ${ }^{9}$ Nakamura et al described uniportal facet-preserving trans-Kambin endoscopic fusion using a standard transforaminal lumbar interbody fusion (TLIF) cage and a modified retractor system. ${ }^{10}$

Several other authors have described similar endoscopic fusion through Kambin's triangle over the past 2 decades, using various terminology such as percutaneous transforaminal endoscopic lumbar interbody fusion, ${ }^{11,12}$ percutaneous endoscopic TLIF, ${ }^{13-15}$ full endoscopic lumbar interbody fusion, ${ }^{16}$ full endoscopic TLIF, ${ }^{17}$ and LEW-LIF. ${ }^{9}$ All the various nomenclature involved insertion of an interbody cage through Kambin's triangle using a transforaminal endoscopic approach and fluoroscopic assistance. Hence, the term trans-Kambin triangle lumbar interbody fusion, which Sariyo et al abbreviated KLIF, is used to describe the aforementioned techniques. ${ }^{18,19}$ We believed that KLIF was an appropriate abbreviation to distinguish the technique from uniportal facet-sacrificing posterolateral TLIF. $^{3,20,21}$

The advantages of introducing an interbody cage through Kambin's triangle with facet preservation or additional foraminoplasty highlighted in the literature are (1) the ability to perform the procedure under local anesthesia and sedation, (2) preservation of the posterior column of the spinal segment, (3) the possibility of direct visual inspection of end plate preparation, and (4) the benefits of minimally invasive spine surgery such as shorter inpatient stay, less perioperative need of opioids, and less blood loss. Commonly described disadvantages of uniportal facet-preserving KLIF are (1) increased incidence of subsidence, especially in a standalone cage, (2) exiting nerve root dysesthesia, likely secondary to nerve root impingement due to the limited working space of Kambin's triangle during the insertion of the cage, (3) heavy reliance on intraoperative fluoroscopy, and (4) the lack of direct decompression of neural structures.

\section{Uniportal Facet-Sacrificing Posterolateral TLIF}

The increased incidence of exiting nerve root dysesthesia and subsidence of small footprint cages in uniportal facet-preserving KLIF drew criticism from open and tubular minimally invasive spine surgeons. Jacquot and Gastambide had a 36\% complication rate in their series, and hence they did not recommend general indication of uniportal facet-preserving KLIF to surgically fit patients; it was only indicated for patients with difficult comorbidities pending decisive technical improvements. ${ }^{15}$ These limitations of uniportal facet-preserving KLIF were inherent to the limited space provided by Kambin's triangle, even with foraminoplasty. Complete facetectomy would provide more working space between the exiting nerve root and traversing nerve root to minimize nerve root retractions and provide sufficient room for larger cage insertion.

Surgeons can overcome space limitations by performing a technique similar to open TLIF, as described by Harms, in which facet resection is performed to optimize the working area adjacent to the disc between the exiting nerve root and traversing nerve root. ${ }^{22} \mathrm{~A}$ similar surgical corridor using the minimally invasive tubular approach TLIF (MIS-TLIF) was first described by Foley et al. ${ }^{23}$ Similar MIS-TLIF approaches had achieved short- to medium-term clinical success in unilateral biportal endoscopy (UBE) and are termed unilateral biportal endoscopic transforaminal lumbar interbody fusion. ${ }^{24,25}$ Surgeons performing UBE can introduce larger equipment through a separate working portal, hence overcoming the technical difficulty in complete facetectomy and obtaining enough autograft for fusion. However, it would be technically challenging to perform complete facetectomy and obtain enough bone graft using a small working channel transforaminal or interlaminar endoscope designed for discectomy.

The development of uniportal facet-sacrificing posterolateral TLIF aimed to achieve the same objectives as MIS-TLIF and unilateral biportal endoscopic transforaminal lumbar interbody fusion through the use of a larger working channel uniportal stenosis endoscope. This development was made possible in late 2010s with growing endoscopic technical expertise and larger working channel uniportal endoscopes designed to perform spinal 
stenosis decompression. ${ }^{26,27}$ The larger working channel uniportal stenosis endoscope allows a larger radiofrequency ablator, endoscopic drill, and endoscopic osteotome to pass through to target tissue, making it possible to perform various spinal decompressions and facet resections in UBE. ${ }^{28}$ Uniportal facet-sacrificing TLIF achieves posterolateral decompression of neural elements, complete facet resection, and insertion of a large interbody cage using a uniportal stenosis endoscope.

The literature on this endoscopic interbody fusion technique is limited; the first description of this subtype of uniportal endoscopic fusion was a case report by Kim et al, in which they reported successfully reducing a grade 2 isthmic spondylolisthesis, performing direct decompression of neural elements and facetectomy, and introducing a traditional TLIF cage using a uniportal facet-sacrificing posterolateral TLIF technique with good clinical outcomes. ${ }^{20} \mathrm{Wu}$ et al subsequently reported the first large retrospective case series using this technique and introduced the term uniportal facet-sacrificing posterolateral TLIF. Their technical note described the application of this technique for patients with high-grade foraminal stenosis secondary to severe collapsed disc space. There were no cases of exiting nerve root dysesthesia in their series. ${ }^{3}$ Kim et al subsequently reported the use of uniportal facet-sacrificing posterolateral TLIF in the treatment of low-grade adult degenerative scoliosis and introduced the concept of safe cage insertion with a Harrison cage glider. ${ }^{21}$ The reported advantages of uniportal facet-sacrificing posterolateral TLIF are (1) direct endoscopic decompression of the neural elements, (2) extension of the working area by facet removal, creating sufficient space for the large TLIF cage used in open surgery to be inserted endoscopically, (3) direct visualization of the end plate during disc preparation, and (4) the known benefits of minimally invasive surgery. The disadvantages are (1) the steep learning curve associated with this surgery, (2) the need for general or epidural anesthesia rather than local anesthesia, and (3) the potential risks to the traversing nerve root during cage insertion, necessitating a specialized cage glider (Table).

\section{BRIEF TECHNICAL DESCRIPTION}

\section{Uniportal Facet-Preserving KLIF}

Various versions of this technique were described in the preceding section, and the specific technique we used is descibed in the following. Reference can be made to recent work by Sairyo et al. ${ }^{18}$

The patient is placed in the prone position under either general anesthesia, epidural anesthesia with sedation, or local anesthesia with sedation on a radiolucent table. Neuromonitoring is performed with free-running electromyography in general anesthesia cases. The table is adjusted so that the caudal vertebral body end plate is parallel to the beam of fluoroscopy. Spondylolisthesis is reduced and disc height is extended prior to uniportal facetpreserving KLIF insertion. The working cannula of the transforaminal endoscope with a working length of $171 \mathrm{~mm}$, a $6.3-\mathrm{mm}$ outer diameter, a working channel diameter of $3.7 \mathrm{~mm}$, and an optical angle of $30^{\circ}$ is docked on the superior articular process, and foraminoplasty is then performed using endoscopic vision or fluoroscopic guidance, according to the surgeon's preference (Figure 3A). A 12-mm depth of disc space is created for safe insertion of an expandable cage. This disc space preparation can be performed using endoscopic vision by drilling $12 \mathrm{~mm}$ of disc space, which is roughly 4 times the length of the 3-mm endoscopic surgical drill inserted under fluoroscopic guidance to the center of the disc. A blunt guidewire is then inserted into the center of the disc through the endoscope, and the endoscope is removed. Serial dilation is performed

Table. Differences between uniportal facet-preserving trans-Kambin endoscopic fusion and uniportal facet-sacrificing posterolateral transforaminal lumbar interbody fusion.

\begin{tabular}{|c|c|c|}
\hline Characteristics & $\begin{array}{l}\text { Uniportal Facet-Preserving Trans-Kambin } \\
\text { Endoscopic Fusion }\end{array}$ & $\begin{array}{l}\text { Uniportal Facet-Sacrificing Posterolateral } \\
\text { Transforaminal Lumbar Interbody Fusion }\end{array}$ \\
\hline Approach & Kambin's triangle & Extended Kambin's triangle \\
\hline Size of Endoscope & Uniportal transforaminal endoscope & Uniportal stenosis endoscope \\
\hline Type of Cage & Expandable cage, OptiMesh, modified TLIF cages & Open or MIS-TLIF cage \\
\hline Handling of Facet & $\begin{array}{l}\text { Preserved or foraminoplasty } \\
\text { Not from lumbar segment; obtained from iliac crest }\end{array}$ & Complete facetectomy \\
\hline Availability of Autograft & or other sources & Obtained from bony decompression and facet \\
\hline Neural Decompression & Indirect decompression & Direct decompression \\
\hline Method of Anesthesia & Local anesthesia, epidural, general anesthesia & Epidural, general anesthesia \\
\hline
\end{tabular}

Abbreviations: MIS-TLIF, minimally invasive transforaminal lumbar interbody fusion; TLIF, transforaminal lumbar interbody fusion. 


\section{Disc space widening with the opening devices}

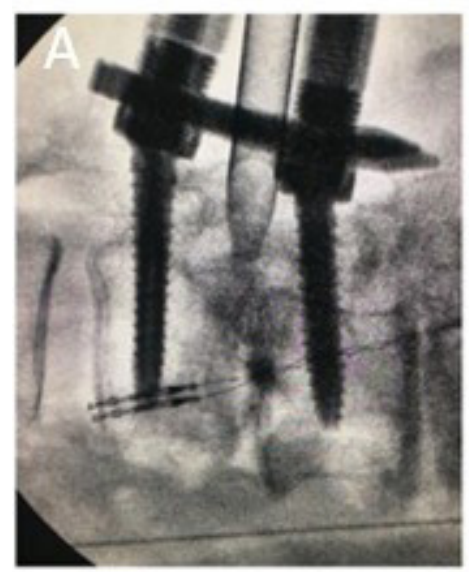

Before

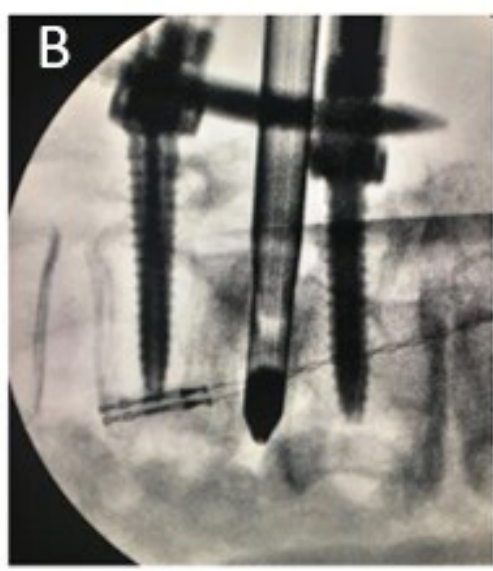

$8 \mathrm{~mm}$ Cannula

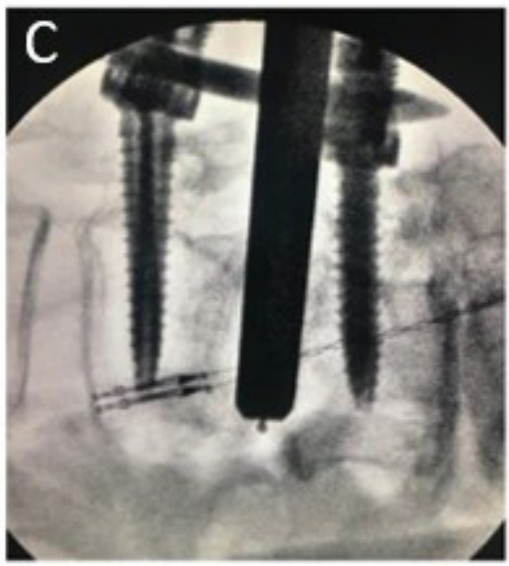

$10 \mathrm{~mm}$ Open square cannula

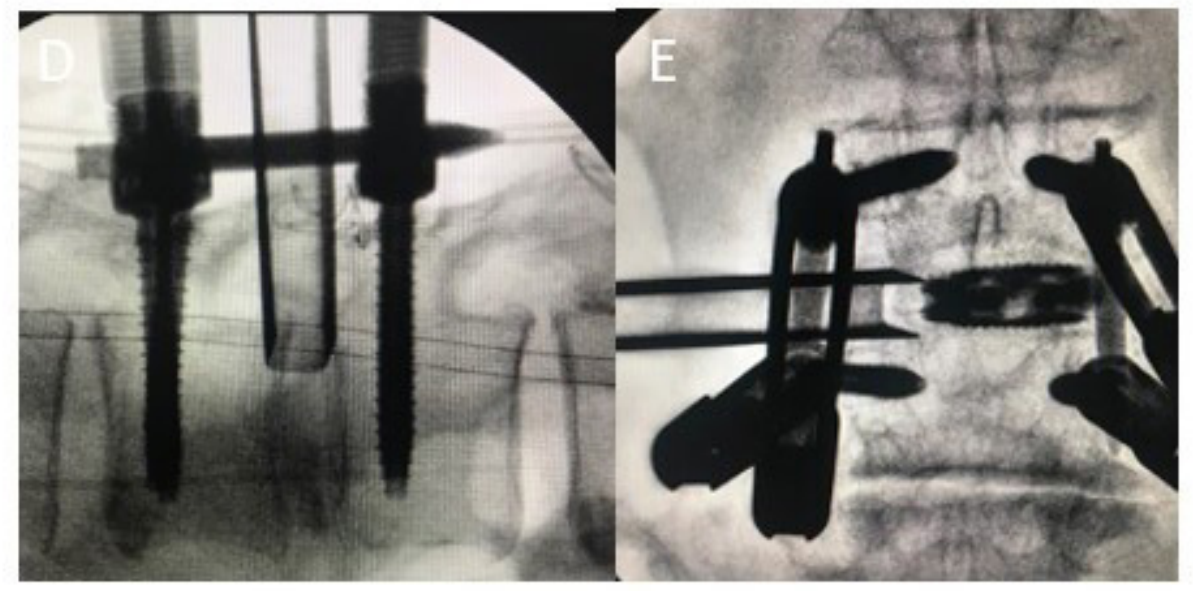

Figure 3. Intraoperative fluoroscopic pictures of uniportal facet-preserving trans-Kambin endoscopic fusion. (A) The working cannula of the transforaminal endoscope is docked in Kambin's triangle directly on the disc space. Foraminoplasty is optional, depending on the size of Kambin's triangle and the presence of exiting nerve root irritation. (B) 8-mm dilation with cannula inserted. (C) 10-mm dilation with open square cannula inserted. (D) Lateral fluoroscopic view of the open square cage glider. (E) Anteroposterior view of the expandable cage inserted under the protection of an open cage glider. Figure reproduced with permission from Sairyo K, Morimoto M, Yamashita Y, et al. Full-endoscopic trans-Kambin's triangle lumbar interbody fusion: technique and review of literature. J Minim Invasive Spine Surg Tech. 2021;6(Suppl 1):S123-S129.

with a cannulated spacer dilator, inserted at $8 \mathrm{~mm}$ (Figure 3B) and gradually widened to the height of $10 \mathrm{~mm}$. The open square cannula is then inserted and docked just inside the disc to protect the exiting and traversing nerve roots (Figure 3C). The angle of cage insertion should be parallel to the disc on both the anteroposterior and lateral view, with close monitoring of the exiting nerve root electromyography changes in general anesthesia cases or patients' symptoms in local anesthesia cases (Figure 3D). A specialized curette is then inserted to prepare the disc end plate. The endoscope is reintroduced to inspect the adequacy of end plate preparation. Once end plate preparation is satisfactory, the endoscope is removed and the bone graft funnel is inserted. The bone graft is packed into the disc space under fluoroscopic guidance. An expandable cage packed with autogenous bone graft is inserted under fluoroscopic 
guidance through the specialized open square cannula and expanded to the appropriate height (Figure 3E). The endoscope is then reintroduced to examine the final position of the cage and the status of neural decompression. A final check is performed with intraoperative fluoroscopy to confirm the interbody cage and screw positions. ${ }^{18}$

\section{Uniportal Facet-Sacrificing Posterolateral TLIF}

Kim et al described a case of left L4/5 grade 1 spondylolisthesis, which is used to highlight this technique. ${ }^{20}$

The patient is positioned prone in a Wilson frame and radiolucent table under general or epidural anesthesia. A 1.3-cm incision is made on the superior border of the caudal pedicle (left L5) in line with the disc space on lateral fluoroscopy. Serial dilation is performed, and a working cannula with a 13-mm outer diameter for a uniportal stenosis endoscope with a $15^{\circ}$ optical angle, $10-\mathrm{mm}$ outer diameter, and 6-mm working channel is docked on the isthmus of L4. Soft tissue is dissected with a large radiofrequency ablator with a 5-mm diameter to expose the spinolaminar junction, isthmus, and facet joint. The facet joint is released with a small 2-mm radiofrequency ablator. Complete inferior facetectomy is performed by endoscopic drilling of the isthmus to the lateral margin of the superior articular facet, from Wu's point to Kim's point in the inside-out approach or from Kim's point to Wu's point in the outside-in approach. Wu's point is the midpoint of the bony arch that forms from the ipsilateral spinolaminar junction of the cephalad lamina to the most inferomedial rounded edge of the inferior articular process. Kim's point is the confluence of the superolateral edge of the inferior articular facet and the superolateral edge of the superior articular facet. ${ }^{29}$ The inferior articular facet is removed en bloc or piecemeal by endoscopic forceps. The superior articular facet is removed using a Kerrison punch and endoscopic drill on the base of the superior articular facet. The ligamentum flavum is removed with a Kerrison punch and bent probe. Contralateral decompression is optional and generally recommended in patients who have severe central and bilateral recess stenosis, but it is not necessary in unilateral lateral recess stenosis or foraminal stenosis. If contralateral decompression is performed over the top of the ligamentum flavum, sublaminar bony decompression is performed with an endoscopic drill. Once bony decompression reaches the margins of the ligamentum flavum attachment, the ligamentum flavum will be loose and can be retrieved with endoscopic forceps and a Kerrison punch. The ipsilateral traversing nerve root is protected and gently retracted using a beveled 13-mm working cannula with the opening pointing away from the traversing nerve root, and discectomy is performed using a blunt probe and endoscopic forceps. The tip of bevel is then advanced to the posterior vertebra border inside the disc space, and discectomy and end plate preparation are performed with an endoscopic drill, a bent probe, and forceps. The end plate is denuded of cartilage and disc, with punctate bleeding observed under endoscopic inspection. The endoscope is removed, and a dilator is placed in the disc space through the

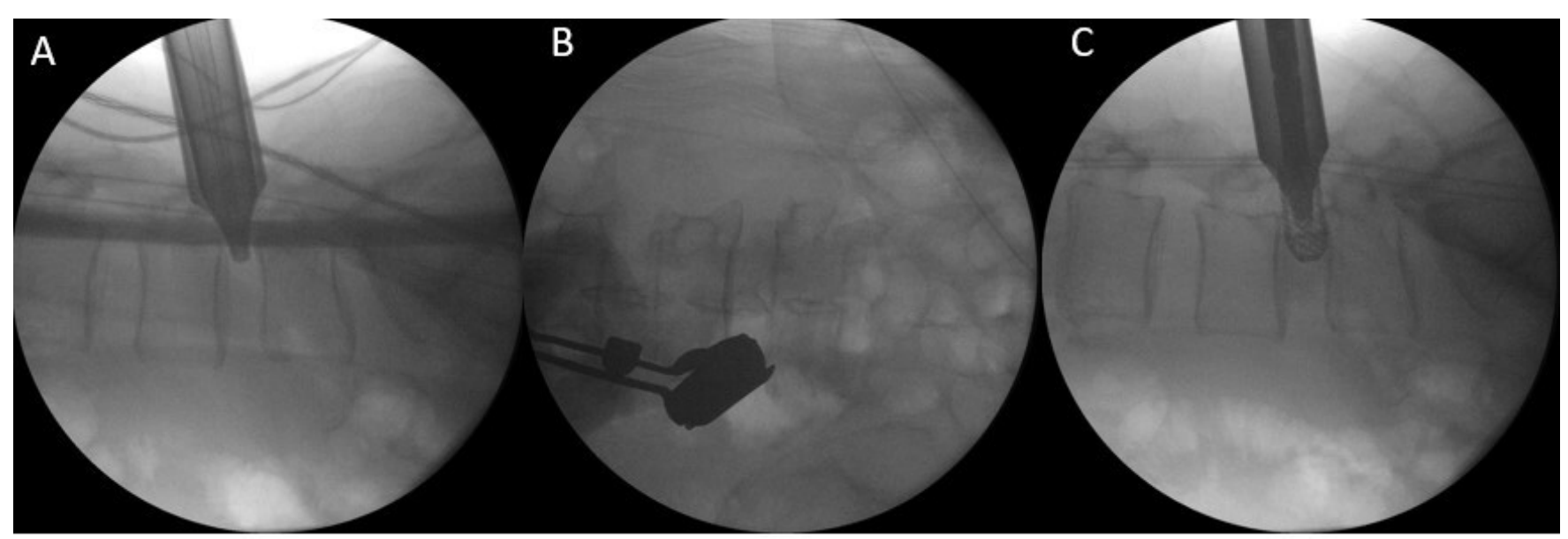

Figure 4. Intraoperative fluoroscopic pictures of uniportal facet-sacrificing posterolateral transforaminal lumbar interbody fusion. (A) Lateral fluoroscopic view of the Harrison cage glider put in place with the tip of the cage glider in the posterior third of the disc space. The cage glider protects the traversing and exiting nerve root. (B) Anteroposterior fluoroscopic view of the Harrison cage glider with the tip positioned in the medial and lateral parts of the disc space, protecting the traversing and exiting nerve root. (C) Lateral fluoroscopic view of the interbody cage being inserted through the Harrison cage glider. 
beveled working cannula, with the traversing nerve root protected (Figure 4A,B). Fluoroscopic-guided dilation and trial are performed, and the disc space is packed with autogenous bone graft through a typical bone graft funnel available on any TLIF equipment tray. An appropriately sized interbody cage packed with autogenous bone graft is inserted under fluoroscopic guidance (Figure 4C). The endoscope is then reintroduced to examine the final position of the cage and the status of the neural decompression. After cage insertion, percutaneous pedicle screws are inserted in standard fashion. We typically insert pedicle screws after cage insertion. ${ }^{3}$

\section{INDICATIONS FOR ENDOSCOPIC FUSION}

Both uniportal facet-preserving KLIF and uniportal facet-sacrificing posterolateral TLIF have similar indications to open TLIF and MIS-TLIF. Common indications described in the literature are spondylolisthesis, spinal instability with degenerative disc disease, spinal stenosis with both mechanical back and leg pain, foraminal stenosis, and recurrent disc herniations in patients who have undergone failed conservative management. ${ }^{3,9,30-32}$

Uniportal facet-preserving KLIF is especially helpful in patients who have severe heart and lung conditions and are at high risk of general anesthesia-related complications. In such patients, uniportal facet-preserving KLIF is performed with local anesthesia under monitored sedation (Figure 5). ${ }^{15}$

Extended indications of uniportal facet-sacrificing posterolateral TLIF to adult low-grade Cobb angle degenerative scoliosis for short segment fusion have been described. ${ }^{21}$ For single-level fusion, uniportal facet-preserving KLIF can be performed under local anesthesia and monitored sedation. For single- and multilevel fusions, uniportal facet-sacrificing posterolateral TLIF can be performed under general anesthesia (Figure 6).

\section{Relative Contraindications for Fusion}

Contraindications for uniportal facet-preserving KLIF are not absolute, as experts who have overcome the learning curve associated with the technique could also overcome the contraindications with additional procedures.

Relative contraindications are:

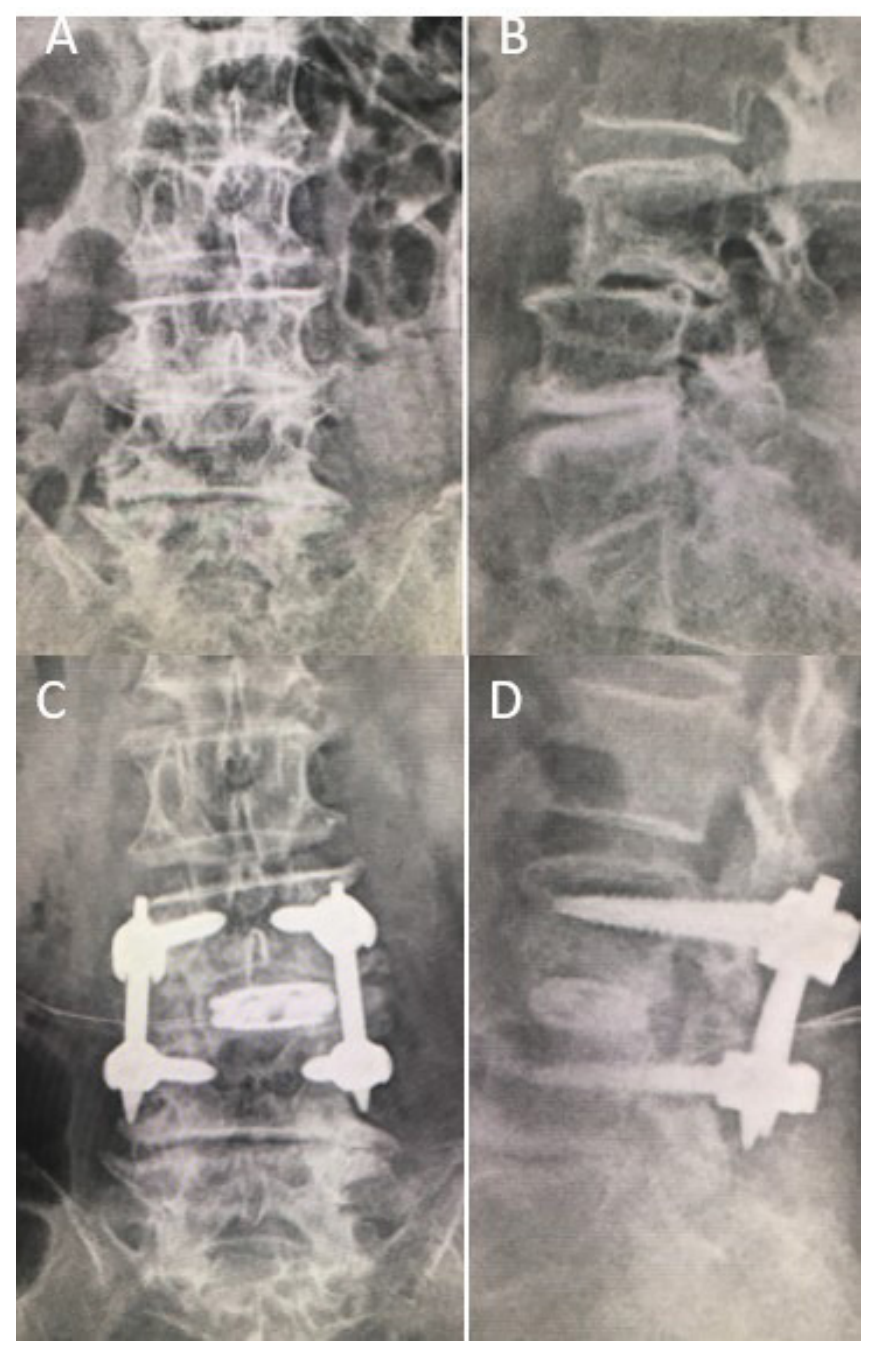

Figure 5. Pre- and postoperative X-ray for single-level L3/4 uniportal facetpreserving trans-Kambin endoscopic fusion performed on an 84-year-old female who presented with $L 3 / 4$ spondylolisthesis and $L 3 / 4$ foraminal stenosis with back and leg pain. $(A, B)$ Preoperative anteroposterior and lateral views of the patient showing the retrolisthesis of L3 on L4 and foraminal stenosis. (C,D) Corresponding postoperative anteroposterior and lateral views of the patient showing restoration of disc height with expansion of foraminal dimension and correction of the retrolisthesis to a neutral position.

1. Nerve root anomalies such as a conjoined root or an additional nerve root in the exit neural foramen. $^{33}$

2. Severe foraminal stenosis where there is a risk of postoperative exiting nerve root dysesthesia. In such cases, careful endoscope-assisted foraminoplasty with the mobile outside-in technique is needed before disc preparation. ${ }^{34}$

3. Severe central canal stenosis and contralateral lateral recess stenosis. ${ }^{35}$ While ipsilateral lateral recess stenosis can be decompressed with extended uniportal facet-preserving KLIF in expert hands, ${ }^{31}$ contralateral lateral recess stenosis requires additional surgical procedures to decompress 


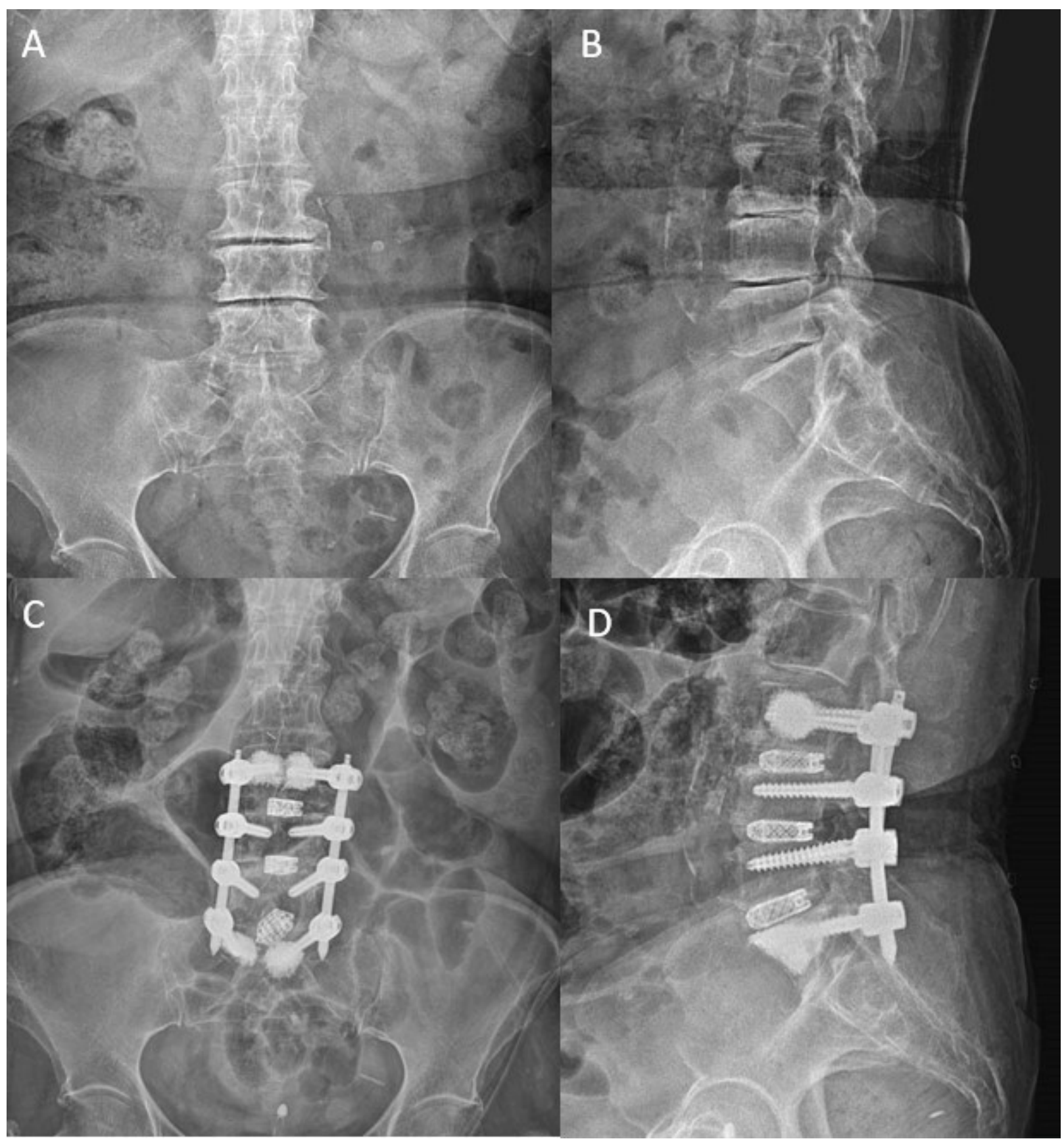

Figure 6. Pre- and postoperative X-ray for 3-level (L3-S1) uniportal facet-sacrificing posterolateral transforaminal lumbar interbody fusion performed on a 70-yearold female who presented with L3-S1 spinal stenosis with back and leg pain. (A,B) Preoperative anteroposterior and lateral views of the patient showing significant loss of disc height, foraminal stenosis, and vacuum signs on L3-S1. (C,D) Corresponding postoperative anteroposterior and lateral views of the patient showing restoration of disc height with expansion of the foraminal dimension and improvement in sagittal alignment after 3-level uniportal facet-sacrificing posterolateral transforaminal lumbar interbody fusion.

with a separate approach. This limitation can be overcome by performing uniportal facetpreserving KLIF from both sides. ${ }^{10}$

4. Severe osteoporosis, which introduces the risk of subsidence in uniportal facet-preserving KLIF with relatively smaller footprints and expandable cages. $^{8}$
5. High iliac crest with L5-S1 level endoscopic fusion.

6. Multiple fusion levels. If local anesthesia and sedation are used, we recommend performing single-level uniportal facet-preserving KLIF rather than multilevel uniportal facet-preserving KLIF because of the expected length of the procedure 
beyond the working time of local anesthesia. As anesthetists are generally uncomfortable managing the airway when a patient is in the prone position, the procedure must be performed expeditiously with a general cutoff time of 120 minutes. ${ }^{8}$ Additional indications for multilevel uniportal facet-preserving KLIF are subject to the surgeon's mastery of the learning curve.

\section{Relative Contraindications for Both Uniportal Facet-Preserving KLIF and Uniportal Facet- Sacrificing Posterolateral TLIF}

The relative contraindications for both uniportal facet-preserving KLIF and uniportal facet-sacrificing posterolateral TLIF include (1) obesity (BMI $>40$ ), as the endoscopic instruments might not be long enough to reach the target site; (2) severe coronal, sagittal, and translation deformity where more bony releases are required $^{21}$; (3) high-grade spondylolisthesis (grades 3 and 4); (4) revision surgery when previous surgery required an open approach for removal and adjustment of instrumentation; and (5) nondegenerative spinal conditions that require fusion, such as infection, tumor, metabolic bone disease, congenital spinal disorders, and trauma.

\section{Relative Contraindications for Uniportal Facet-Sacrificing Posterolateral TLIF}

Uniportal facet-sacrificing posterolateral TLIF deploys a similar approach to MIS-TLIF. The relative contraindications are the same as those for fusion. Patients who are unfit for general anesthesia are unfit for uniportal facet-sacrificing posterolateral TLIF; hence, uniportal facet-preserving KLIF is a better option for these patients, as suggested by Jacquot and Gastambide. ${ }^{15}$ Patients who have a tendency to bleed because of their medical conditions or medications are contraindicated for endoscopic procedures, especially uniportal facet-sacrificing posterolateral TLIF, as significant bony decompression and facet resection are needed.

The main limitation for both types of uniportal endoscopic fusion is the significant learning curve. We recommend that endoscopic surgeons who will perform uniportal facet-preserving KLIF be familiar with transforaminal lumbar endoscopic foraminal decompression. Surgeons who would like to perform uniportal facet-sacrificing posterolateral TLIF should be familiar with lumbar endoscopic unilateral laminotomy for bilateral decompression, as endoscopic drills are used extensively. ${ }^{36}$

\section{CLINICAL OUTCOMES}

In a recently published meta-analysis by $\mathrm{Heo}$ et al, analysis of pre- and postoperative Oswestry Disability Index (ODI) scores and visual analog scale (VAS) scores for back and leg pain was performed for 13 studies that met the inclusion and exclusion criteria. They set the minimal clinically important difference (MCID) for VAS to 3 points and the MCID for ODI to 15 points to indicate significant improvement after endoscopic fusion. ${ }^{24}$ They found ODI scores to have twice the MCID for improvement, and both VAS leg and back scores met MCID requirements. ${ }^{24}$ Fusion rates range from $85 \%$ to $100 \%$ in uniportal endoscopic fusion. $^{8,12,13,37,38}$ The proposed benefits are direct docking and access, as with minimally invasive surgery, and optimized visualization of the target tissues with limited collateral soft tissue damages. The delivery of instruments through the working channel has the advantage of limited repetitive soft tissue trauma to collateral soft tissues adjacent to the target disc.

Patients who undergo endoscopic fusion have a postoperative recovery marked by decreased VAS back pain scores, less reliance on opioids, early mobilization, and a shortened hospital stay. In a recent publication by Sairyo et al, there was one case of transient exiting nerve root irritation at L4 following L4/5 uniportal facet-preserving KLIF, with no other subsequent complications. There was statistically significant improvement of both VAS back and leg pain scores (6.9 to 0.9 and 6.0 to 0.9 , respectively, $P<0.05) .{ }^{18} \mathrm{Kim}$ et $\mathrm{al}^{21}$ performed a retrospective case control study of clinical and computer tomographic fusion and subsidence evaluation for single-level uniportal endoscopic posterolateral TLIF versus microscopic minimally invasive transforaminal interbody fusion. They compared 33 levels of uniportal facet-sacrificing posterolateral TLIF with 22 levels of TLIF and found that both techniques achieved significant improvement in postoperative pain and ODI scores at 4 weeks, 3 months, and final follow-up, with VAS scores of $4.39 \pm 0.92,5.27 \pm 1.16$, and $5.73 \pm 1.21$ for uniportal facet-sacrificing posterolateral TLIF and $4.55 \pm 1.16,5.05 \pm 1.11$, and $5.50 \pm 1.20$ for TLIF, respectively; postoperative ODI scores at 1 week, 3 months, and final follow-up were $43.15 \pm 6.57,49.27$ \pm 8.24 , and $51.73 \pm 9.09$ for uniportal facet-sacrificing posterolateral TLIF and 41.73 $\pm 7.98,46.18 \pm 8.46$, and $49.09 \pm 8.98$ for TLIF, respectively $(P<0.05)$. Compared to TLIF, uniportal facet-sacrificing posterolateral TLIF achieved better VAS and ODI scores, with 0.727 \pm 0.235 and $3.88 \pm 1.50$ at 3 months and $0.727 \pm 0.252$ and $3.42 \pm 1.63$ at final follow-up, respectively $(P<$ 
0.05). At 6 months, radiological evaluation comparing the fusion techniques showed a significantly more favorable fusion rate with uniportal facet-sacrificing posterolateral TLIF (Bridwell grade of $0.61 \pm 0.12$ at 6 months and $0.49 \pm 0.12$ at 1 year; Kim stage of $0.70 \pm$ 0.15 at 6 months and $0.56 \pm 0.14$ at 1 year). There was also less subsidence, with a Kim's subsidence grade of $0.606 \pm 0.18$ at 6 months and $0.561 \pm 0.20$ at 1 year $(P$ $<0.05){ }^{38}$

Overall, both uniportal facet-preserving KLIF and uniportal facet-sacrificing posterolateral transforaminal lumbar interbody fTLIFusion showed promising clinical outcomes comparable to those of other fusion methods. In expert hands, they delivered good results with the least invasive method of fusion, hence maximizing perioperative experience and outcomes.

\section{COMPLICATIONS}

Most complications of endoscopic fusion are minor and can usually be resolved with conservative treatment or revision surgery. There were no major complications reported in the literature, and no significant difference in complication rates between conventional TLIF, MISTLIF, and endoscopic fusion. ${ }^{37}$

\section{General Complications of Spinal Fusion}

Inherent complications of spinal fusion are malposition of screws and cage, implant breakage and failure, loosening screws, cage subsidence, nonunion, and cage migration. ${ }^{39}$

\section{Complications Specific to Endoscopy}

Water pressure-related complications such as postoperative headache, neck pain, and seizures can be managed with careful titration of irrigation fluid pressure to $30-50 \mathrm{mmHg}$ during most of the procedure. ${ }^{40}$ Incidental durotomy during endoscopic decompression can be managed with patch blocking repairs or anastomotic clips. ${ }^{41}$ Postoperative hematoma is inherent to all endoscopic and open procedures. Meticulous hemostasis, careful avoidance of patients with bleeding diatheses, and the usage of a drain can decrease the risk of postoperative hematoma. ${ }^{42}$

\section{Complications Specific to Uniportal Facet- Preserving KLIF}

Exiting nerve root injury and subsidence are the most critical complications of uniportal facet-preserving KLIF. The inherent limited working space of Kambin's triangle can cause the exiting nerve root to get caught between the unyielding cephalad vertebra pedicle and the metal working cannula, leading to significant postoperative dysesthesia. ${ }^{15,43}$ Foraminoplasty can help create more working space in Kambin's triangle. Neuromonitoring, in general anesthesia cases, or light local anesthesia and monitored sedation, as well as the use of specifically designed cage gliders during cage insertion, can help decrease the rate of exiting nerve root dysesthesia. Cage subsidence has multiple causative factors such as osteoporosis, end plate violation during disc preparation, small footprint end plate, overdistraction of the expandable cage, and use of a standalone cage without pedicle screws. ${ }^{8,9,15}$ Some uniportal facetpreserving KLIF surgeons harvest iliac crest bone graft to provide the autograft required for endoscopic fusion. Inherent donor site morbidity and possible complications are associated with this technique. ${ }^{10}$

\section{Complications Specific to Uniportal Facet- Sacrificing Posterolateral TLIF}

Uniportal facet-sacrificing posterolateral TLIF is performed in close proximity to the traversing nerve root, and thus retraction of the traversing nerve root medially is often required to generate a better angle and create more working space for interbody cage insertion. Incidental durotomy and injuries to the traversing nerve root are a possible complication related to this approach. An appropriately large working cannula and specific cage gliders, such as the Harrison cage glider, can decrease the incidence of nerve root injury. ${ }^{21,44}$

\section{DIFFERENCE BETWEEN BIPORTAL ENDOSCOPIC FUSION AND UNIPORTAL ENDOSCOPIC FUSION}

While the main focus of this article is uniportal endoscopic fusion techniques, it is important to note that UBE fusion is an alternative method of endoscopic fusion that is also gaining traction among spine surgeons who practice unilateral biportal endoscopy. There are significant differences between uniportal and biportal endoscopy in terms of technique and the handling of soft tissue. ${ }^{27,44}$ Heo et al showed significant improvement in VAS and ODI scores with minor perioperative complications in both uniportal and biportal endoscopic techniques. There are no significant differences in early and medium-term postoperative outcomes between fusion techniques. ${ }^{24}$ Overall, the early clinical results of both biportal and uniportal endoscopic fusion approaches are promising, but long-term outcomes 
should be investigated and randomized controlled trials should be conducted.

\section{CONCLUSION}

Uniportal endoscopic fusion is perhaps the least invasive method of spinal fusion reported in the literature. Endoscopic fusion is a double-edged sword, in that it maximizes the benefits of minimally invasive spinal surgery with improved postoperative clinical outcomes, but there is a steep learning curve, limiting its propagation and general use in the spinal community. Despite its early stage of development, there is great potential in this method of fusion.

\section{REFERENCES}

1. Kambin P, Sampson S. Posterolateral percutaneous suctionexcision of herniated lumbar intervertebral discs. Clin Orthop Relat Res. 1986;207:37. doi:10.1097/00003086-198606000-00008.

2. Tumialán LM, Madhavan K, Godzik J, Wang MY. The history of and controversy over Kambin's triangle: a historical analysis of the lumbar transforaminal corridor for endoscopic and surgical approaches. World Neurosurg. 2019;123:402-408. doi:10.1016/j. wneu.2018.10.221.

3. Wu PH, Kim HS, Lee YJ, et al. Uniportal full endoscopic posterolateral transforaminal lumbar interbody fusion with endoscopic disc drilling preparation technique for symptomatic foraminal stenosis secondary to severe collapsed disc space: a clinical and computer tomographic study with technical nFull Endoscopic Posterolateral Transforaminal Lumbar Interbody Fusion with Endoscopic Disc Drilling Preparation Technique for Symptomatic Foraminal Stenosis Secondary to Severe Collapsed Disc Space: A Clinical and Computer Tomographic Study with Technical Note. Brain Sci. 2020;10(6):E373. doi:10.3390/brainsci10060373.

4. Kim M, Kim H-S, Oh SW, et al. Evolution of spinal endoscopic surgery. Neurospine. 2019;16(1):6-14. doi:10.14245/ ns.1836322.161.

5. Leu HF, Hauser RK. Percutaneous endoscopic lumbar spine fusion. Neurosurg Clin N Am. 1996;7(1):107-117.

6. Osman SG. Endoscopic transforaminal decompression, interbody fusion, and percutaneous pedicle screw implantation of the lumbar spine: A case series report. Int J Spine Surg. 2012;6:157-166. doi:10.1016/j.ijsp.2012.04.001.

7. Morgenstern R, Morgenstern C, Jané R, Lee S-H. Usefulness of an expandable interbody spacer for the treatment of foraminal stenosis in extremely collapsed disks: preliminary clinical experience with endoscopic posterolateral transforaminal approach. J Spinal Disord Tech. 2011;24(8):485-491. doi:10.1097/ BSD.0b013e3182064614.

8. Wang MY, Grossman J. Endoscopic minimally invasive transforaminal interbody fusion without general anesthesia: initial clinical experience with 1-year follow-up. Neurosurg Focus. 2016;40(2):E13. doi:10.3171/2015.11.FOCUS15435.

9. Lewandrowski K-U, Ransom NA, Ramírez León JF, Yeung A. The concept for a standalone lordotic endoscopic wedge lumbar interbody fusion: the LEW-LIF. Neurospine. 2019;16(1):82-95. doi:10.14245/ns.1938046.023.
10. Nakamura S, Ito F, Ito Z, Shibayama M. Methods and early clinical results of percutaneous lumbar interbody fusion. Neurospine. 2020;17(4):910-920. doi:10.14245/ns.2040302.151.

11. Lee SH, Erken HY, Bae J. Percutaneous transforaminal endoscopic lumbar interbody fusion: clinical and radiological results of mean 46-month follow-up. 2017;2017:3731983. doi:10.1016/j. ijsu.2017.04.053.

12. Wu J, Liu H, Ao S, et al. Percutaneous endoscopic lumbar interbody fusion: technical note and preliminary clinical experience with 2-year follow-up. Biomed Res Int. 2018;2018. doi:10.1155/2018/5806037.

13. Yang J, Liu C. Percutaneous endoscopic transforaminal lumbar interbody fusion for the treatment of lumbar spinal stenosis: preliminary report of seven cases with 12-month follow-up. 2019;2019:3091459. doi:10.31616/asj.2018.0210.

14. Nagahama K, Ito M, Abe Y, Murota E, Hiratsuka S, Takahata M. Early clinical results of percutaneous endoscopic transforaminal lumbar interbody fusion: a new modified technique for treating degenerative lumbar spondylolisthesis. Spine Surg Relat Res. 2019;3(4):327-334. doi:10.22603/ssrr.2018-0058.

15. Jacquot F, Gastambide D. Percutaneous endoscopic transforaminal lumbar interbody fusion: is it worth it? Int Orthop. 2013;37(8):1507-1510. doi:10.1007/s00264-013-1905-6.

16. Youn MS, Shin JK. Full endoscopic lumbar interbody fusion (FELIF): technical note. Aug. 2018;27(8):1949-1955. doi:10.4055/cios.2018.10.2.248.

17. Kamson S, Lu D, Sampson PD, Zhang Y. Full-endoscopic lumbar fusion outcomes in patients with minimal deformities: a retrospective study of data collected between 2011 and 2015. Pain Physician. 2019;22(1):75-88.

18. Sairyo K, Morimoto M, Yamashita K, et al. Full-endoscopic trans-Kambin's triangle lumbar interbody fusion: technique and review of literaopic Trans-Kambin's Triangle Lumbar Interbody Fusion: Technique and Review of Literature. J Minim Invasive Spine Surg Tech. 2021;6(Suppl 1):S123-S129. doi:10.21182/ jmisst.2021.00108.

19. Morimoto M. Full-endoscopic trans-Kambin's triangle lumbar interbody fusion (Fullendo-KLIF). Transforaminal FullEndoscopic Lumbar Surgery under the Local Anesthesia: State of the Art. Springer; 2020:87-95.

20. Kim HS, Wu PH, Jang I-T. Technical note on uniportal full endoscopic posterolateral approach transforaminal lumbar interbody fusion with reduction for grade 2 spondylolisthesis. Interdiscip Neurosurg. 2020;21:100712. doi:10.1016/j.inat.2020.100712.

21. Kim HS, Wu PH, Lee YJ, Kim DH, Jang IT. Technical considerations of uniportal endoscopic posterolateral lumbar interbody fusion: a review of its early clinical results in application in adult degenerative scoliosis. World Neurosurg X. 2021;145:682-692. doi:10.1016/j.wneu.2020.05.239.

22. Harms J. Dorsale repositionsspondylodese bei lumbalen spondylolisthesis. Oper Orthop Traumatol. 1999;11(1):79-79. doi:10.1007/s00064-006-0085-6.

23. Foley KT, Holly LT, Schwender JD. Minimally invasive lumbar fusion. Spine. 2003;28(15 Suppl):S26-35. doi:10.1097/01. BRS.0000076895.52418.5E.

24. Heo DH, Lee DC, Kim HS, Park CK, Chung H. Clinical results and complications of endoscopic lumbar interbody fusion for lumbar degenerative disease: a meta-analysis. World Neurosurgery. 2021;145:396-404. doi:10.1016/j.wneu.2020.10.033.

25. Heo DH, Son SK, Eum JH, Park CK. Fully endoscopic lumbar interbody fusion using a percutaneous unilateral biportal 
endoscopic technique: technical note and preliminary clinical results. Biomed Res Int. 2017;43(2):E8. doi:10.1155/2017/3431257.

26. Kim H-. S, Wu PH, Jang I-T. Lumbar endoscopic unilateral laminotomy for bilateral decompression outside-in approach: a proctorship guideline with 12 steps of effectiveness and safety. Neurospine. 2020;17(Suppl 1):S99-S109. doi:10.14245/ns.2040078.039.

27. Ruetten S. Full-endoscopic operations of the spine in disk herniations and spinal stenosis. Surg Technol Int. 2011;21:284-298.

28. Wu PH, Kim HS, Choi DJ, Gamaliel Y-HT. Overview of tips in overcoming learning curve in uniportal and biportal endoscopic spine surgery. J Minim Invasive Spine Surg Tech. 2021;6(Suppl 1):S84-S96. doi:10.21182/jmisst.2020.00024.

29. Kim H-S, Wu P-H, An J-W, et al. Evaluation of two methods (inside-out/outside-in) inferior articular process resection for uniportal full endoscopic posterolateral transforaminal lumbar interbody fusion: technical note. Brain Sciences. 2021;11(9):1169. doi:10.3390/brainsci11091169.

30. Kim HS, Raorane HD, PH W, et al. Feasibility of endoscopic transforaminal lumbar interbody fusion (eTLIF) through the posterior paraspinal approach: technical note and preliminary result. J Minim Invasive Spine Surg Tech. 2021;6(1):35-41. doi:10.21182/ jmisst.2021.00045.

31. Sairyo K, Yamashita K, Manabe H, et al. A novel surgical concept of transforaminal full-endoscopic lumbar undercutting laminectomy (TE-LUL) for central canal stenosis of the lumbar spine with local anesthesia : A case report and literature review. $J$ Med Invest. 2019;66(3.4):224-229. doi:10.2152/jmi.66.224.

32. Wagner R, Haefner M. Uniportal endoscopic lumbar interbody fusion. Neurospine. 2020;17(Suppl 1):S120-S128. doi:10.14245/ns.2040130.065.

33. Wu PH, Kim HS, Jang I-T. A narrative review of development of full-endoscopic lumbar spine surgery. Neurospine. 2020;17(Suppl 1):S20-S33. doi:10.14245/ns.2040116.058.

34. Kim HS, Adsul N, Kapoor A, et al. A mobile outside-in technique of transforaminal lumbar endoscopy for lumbar disc herniations. J Vis Exp. 2018;(138). doi:10.3791/57999.

35. Lewandrowski K-U, Dowling Á, de Carvalho PST, et al. Indication and contraindication of endoscopic transforaminal lumbar decompression. World Neurosurgery. 2021;145:631-642. doi:10.1016/j.wneu.2020.03.076.

36. Wu PH. Early career challenges in setting up an endoscopic spine surgery practice. World Neurosurgery. 2020;144:264-269. doi:10.1016/j.wneu.2020.09.056.

37. Ao S, Zheng W, Wu J, et al. Comparison of preliminary clinical outcomes between percutaneous endoscopic and minimally invasive transforaminal lumbar interbody fusion for lumbar degenerative diseases in a tertiary hospital: is percutaneous endoscopic procedure superior to MIS-TLIF? A prospective cohort study. Int $J$ Surg. 2020;76:136-143. doi:10.1016/j.ijsu.2020.02.043.

38. Jin M, Zhang J, Shao H, Liu J, Huang Y. Percutaneous transforaminal endoscopic lumbar interbody fusion for degenerative lumbar diseases: a consecutive case series with mean 2-year follow-up. Pain Physician. 2020;23(2):165-174.

39. Kolcun JPG, Brusko GD, Basil GW, Epstein R, Wang MY. Endoscopic transforaminal lumbar interbody fusion without general anesthesia: operative and clinical outcomes in 100 consecutive patients with a minimum 1-year follow-up. Neurosurgical Focus. 2019;46(4):E14. doi:10.3171/2018.12.FOCUS18701.

40. Kang MS, Park HJ, Hwang JH, Kim JE, Choi DJ, Chung HJ. Safety evaluation of biportal endoscopic lumbar discectomy: assessment of cervical epidural pressure during surgery. Spine. 2020;45(20):E1349-E1356. doi:10.1097/BRS.0000000000003585.

41. Kim HS, Raorane HD, Wu PH, Heo DH, Sharma SB, Jang I-T. Incidental durotomy during endoscopic stenosis lumbar decompression: incidence, classification, and proposed management strategies. World Neurosurgery. 2020;139:e13-e22. doi:10.1016/j. wneu.2020.01.242.

42. Zhou C, Zhang G, Panchal RR, et al. Unique complications of percutaneous endoscopic lumbar discectomy and percutaneous endoscopic interlaminar discectomy. Pain Physician. 2018;21(2):E105-E112.

43. Morgenstern C, Yue JJ, Morgenstern R. Full percutaneous transforaminal lumbar interbody fusion using the facet-sparing, trans-Kambin approach. Clin Spine Surg. 2020;33(1):40-45. doi:10.1097/BSD.0000000000000827.

44. Kitahama Y, Sairyo K, Dezawa A. Percutaneous endoscopic transforaminal approach to decompress the lateral recess in an elderly patient with spinal canal stenosis, herniated nucleus pulposus and pulmonary comorbidities. Asian J Endosc Surg. 2013;6(2):130-133. doi:10.1111/ases.12004.

Funding: The author(s) received no financial support for the research, authorship, and/or publication of this article.

Declaration of Conflicting Interests: The authors report no conflicts of interest associated with this work.

Corresponding Author: Hyeun Sung Kim, Department of Neurosurgery, Nanoori Gangnam Hospital, Seoul, South Korea; neurospinekim@gmail. com

Published 31 December 2021

This manuscript is generously published free of charge by ISASS, the International Society for the Advancement of Spine Surgery. Copyright (c) 2022 ISASS. To see more or order reprints or permissions, see http:// ijssurgery.com. 